\title{
The Impact of Adding Taxanes to Anthracyclines on Women with Breast Cancer Receiving Adjuvant Chemotherapy
}

\author{
Erina Hilaj ${ }^{1}$, Alketa Ymeri ${ }^{2}$, Kleva P. Shpati ${ }^{3,4}$ \\ 1. Oncology, National Center of Continuing Education for Health Professionals, Tirana, ALB 2. Oncology, University \\ Hospital Center "Mother Theresa", Tirana, ALB 3. Internal Medicine, Albanian University, Tirana, ALB 4. Internal \\ Medicine, University of Medicine, Tirana, ALB
}

Corresponding author: Erina Hilaj, erinahilaj@yahoo.com

\section{Abstract}

\section{Introduction}

This study aimed to analyze the impact of adding taxanes to anthracycline-based regimens on women diagnosed with breast cancer and treated with adjuvant chemotherapy.

\section{Methods}

This retrospective study included 559 female breast cancer patients who underwent adjuvant chemotherapy at the University Hospital Center “Mother Teresa” in Tirana, Albania from 2005 to 2011. Three hundred fifty-nine patients received an anthracycline-based regimen, and 200 received anthracycline-plus-taxane regimens. Common anthracycline-based regimens consisted of 5 -fluorouracil $600 \mathrm{mg} / \mathrm{m}^{2}$, doxorubicin 60 $\mathrm{mg} / \mathrm{m}^{2}$, cyclophosphamide $600 \mathrm{mg} / \mathrm{m}^{2}$ every three weeks for six cycles. Combined taxane-anthracycline regimens were anthracycline-based regimen in the first four cycles (doxorubicin $60 \mathrm{mg} / \mathrm{m}^{2}$, cyclophosphamide $600 \mathrm{mg} / \mathrm{m}^{2}$, docetaxel $80 \mathrm{mg} / \mathrm{m}^{2}$ ) followed by either weekly paclitaxel or thrice-weekly docetaxel for four cycles.

\section{Results}

Overall, after a 5-year follow-up, it was found that 148 women in the taxanes-based regimen group (74\%) did not experience relapse compared with 264 women in the anthracycline-based regimen group (73.5\%). The relapse status was affected by hormonal status ( $\mathrm{p}:<0.001)$ in the taxane-based regimen. In the anthracycline-based regimen patients, the relapse status was affected by hormone status and nodal involvement ( $\mathrm{p}$ : <0.001).

\section{Conclusion}

Received 02/18/2020 Review began 02/20/2020 Review ended 02/24/2020 Published 02/27/2020

\section{(c) Copyright 2020}

Hilaj et al. This is an open access article distributed under the terms of the Creative Commons Attribution License CC-BY 4.0., which permits unrestricted use, distribution, and reproduction in any medium, provided the original author and source are credited.

The taxanes-plus-anthracycline regimen was slightly more effective than the anthracycline-based regimen for breast cancer patients in terms of avoiding relapse, but the difference was not statistically significant. Therefore, adding taxanes to adjuvant chemotherapy for women diagnosed with breast cancer is not beneficial for every subgroup. Hence, the future of breast cancer therapy remains chemotherapy individualized for each patient for optimal outcomes.

Categories: Oncology, Public Health, Other

Keywords: albania, taxanes-based, breast cancer, chemotherapy.

\section{Introduction}

Breast cancer in Albania is the second-most frequent cancer after lung cancer. Mortality from cancer in Albania is second only to death from circulatory system diseases [1]. Treatment of breast cancer generally involves different modalities, such as surgery, radiation, and chemotherapy. Depending on the model of risk minimization, adjuvant therapy may be responsible for a $35-72 \%$ reduction in the breast cancer mortality rate [2]. Adjuvant chemotherapy is the administration of additional therapy after primary surgery to kill or inhibit micrometastasis [3]. The success of adjuvant chemotherapy in breast cancer was reported in 1976 when Bonadonna et al. published the first report on the efficacy of cyclophosphamide, methotrexate, and fluorouracil (CMF) as adjuvant treatment for node-positive breast cancer [4].

Later trials showed that a treatment similar to CMF but which substituted epirubicin for methotrexate (CEF) was even more effective in disease-free survival (DFS) and overall survival (OS) in premenopausal women with axillary node-positive breast cancer [5]. The advantage of a three-drug combination with anthracycline over CMF was confirmed in an individual patient data meta-analysis of Early Breast Cancer Trialists' Collaborative Group [6]. Altogether, 100,000 patients were included in 123 randomized trials. Standard 
regimens consisted of four cycles of doxorubicin hydrochloride [Adriamycin (Pfizer, New York, NY)] and cyclophosphamide (AC) and cyclophosphamide, Adriamycin, and fluorouracil (CAF) or CEF. The metaanalysis found that anthracycline-based regimens with higher cumulative dosage than standard four-cycle AC were superior to standard CMF (response rate: 0.78 ; : 0.0004).

The taxane chemotherapy class of antimicrotubule anticancer agents has been the most critical addition to the chemotherapeutic armamentarium against cancer in recent decades. Paclitaxel and docetaxel have become the most used agents in adjuvant regimens of breast cancer since their approval following excellent results in treating metastatic breast cancer and many other cancer types [7]. Taxanes are preferred in adjuvant chemotherapy of breast cancer due to their pharmacokinetic profile, consistent positive results, and convenient, intermittent, and brief infusion schedule. They are not subject to cross-resistance with anthracyclines and are more active than commonly used anthracyclines [8]. Many randomized clinical trials were conducted to investigate the role of adding taxanes to anthracyclines. Some of these trials established both OS and DFS, whereas other trials did not show any advantage from adding taxanes [9-19].

In our 2016 study, the relapse rate of the taxanes-plus-anthracycline regimen was $23 \%$, but the study was limited by its small number of cases and a lack of comparator group [20]. We conducted the present study with a larger sample of 200 women along with a traditional chemotherapy comparator group to improve the reliability of our initial findings.

The goal of this study was to analyze the impact of adding taxanes to anthracycline-based regimens on women diagnosed with breast cancer and treated with adjuvant chemotherapy in Albania.

\section{Materials And Methods}

Our study was a retrospective analysis that included 559 Albanian female breast cancer patients who underwent adjuvant chemotherapy at the University Hospital Center "Mother Teresa" in Tirana from 2005 to 2011, with a follow-up of five years. Our study included female patients younger than 70 years with a proven diagnosis of breast cancer who underwent surgery and adjuvant chemotherapy. The study excluded patients with metastatic breast cancer, patients receiving neoadjuvant or non-anthracycline-based chemotherapy, and those whose follow-up was shorter than five years.

The adjuvant chemotherapy regimens for patients in the study were anthracycline-based regimen (CAF) or an anthracycline-plus-taxane regimen (AC/T). The most common CAF regimen consisted of 5 -fluorouracil $600 \mathrm{mg} / \mathrm{m} 2$, doxorubicin $60 \mathrm{mg} / \mathrm{m} 2$, and cyclophosphamide $600 \mathrm{mg} / \mathrm{m} 2$ every three weeks for six cycles. The combined taxane-anthracycline regimen consisted of anthracycline-based regimen in the first four cycles (AC/T; doxorubicin $60 \mathrm{mg} / \mathrm{m} 2$, cyclophosphamide $600 \mathrm{mg} / \mathrm{m} 2$, docetaxel $80 \mathrm{mg} / \mathrm{m} 2$ ) and either weekly paclitaxel or thrice-weekly docetaxel for four cycles.

Hormone receptor assays were performed. Estrogen and progesterone receptors were considered positive if $>1 \%$ of tumor cells showed expression by immunohistochemistry (IHC). Human epidermal growth factor receptor 2 (HER2)-positive patients had a score of 3+ by IHC. Most of the patients with overexpressed HER2+ received adjuvant trastuzumab.

Patients were assigned into two groups based on the type of adjuvant chemotherapy regimen received: anthracycline-based regimen and taxane-based regimen. The primary endpoint of this study was DFS, defined as the time from surgery to disease progression in the form of the local breast (including ductal carcinoma in situ) or nodal recurrence and metastatic disease.

\section{Statistical analysis}

Fisher's exact test was used to compare the distribution of demographic and clinical characteristics of women according to their relapse status (relapse vs. no relapse at the end of the follow-up period) for each chemotherapy regimen (AC/T vs. CAF). We used the Kaplan-Meier method to assess chemotherapeutic effectiveness, and the two groups were compared with the log-rank test for significant changes. P-values less than 0.05 were considered statistically significant. IBM SPSS Statistics for Windows, Version 21.0. (IBM Corp., Armonk, NY) was used for all statistical analyses.

\section{Results}

From January 2005 to December 2011, 559 patients fulfilling the inclusion criteria were included in this study. Overall, there were 200 women treated AC/T regimens and 359 women treated with CAF regimens as adjuvant chemotherapy.

Table 1 presents the distribution of relapse time among women treated with AC/T and CAF. Fifty-two women (26\%) experienced relapse within five years in the AC/T group, and 95 women (26.5\%) experienced relapse within five years in the CAF group. A total of 148 women (74\%) in the $\mathrm{AC} / \mathrm{T}$ group had no relapse during the 5-year follow-up, and 264 women (73.5\%) in the CAF group had no relapse during the same period. 


\section{Cureus}

\begin{tabular}{|l|llll|}
\hline & AC/T & & CAF & \\
\hline Relapse time & $\mathrm{n}$ & $(\%)$ & $\mathrm{n}$ & (\%) \\
\hline$<12$ months & 14 & 7 & 16 & 4 \\
\hline $12-24$ months & 21 & 11 & 29 & 8 \\
$25-36$ months & 10 & 5 & 23 & 6 \\
$37-48$ months & 5 & 3 & 16 & 4 \\
$49-60$ months & 2 & 1 & 11 & 3 \\
$>60$ months & 148 & 74 & 264 & 73.5 \\
Total & 200 & 100 & 359 & 100 \\
\hline
\end{tabular}

TABLE 1: Distribution of relapse time among women treated with $A C / T$ and $C A F$

$\mathrm{AC/T}$ : anthracycline-plus-taxane adjuvant chemotherapy; CAF: anthracycline-based adjuvant chemotherapy

We noted no significant difference in 5-year DFS when comparing CAF with AC/T (P: 0.943). Kaplan-Meier survival analyses are presented in Figure 1.

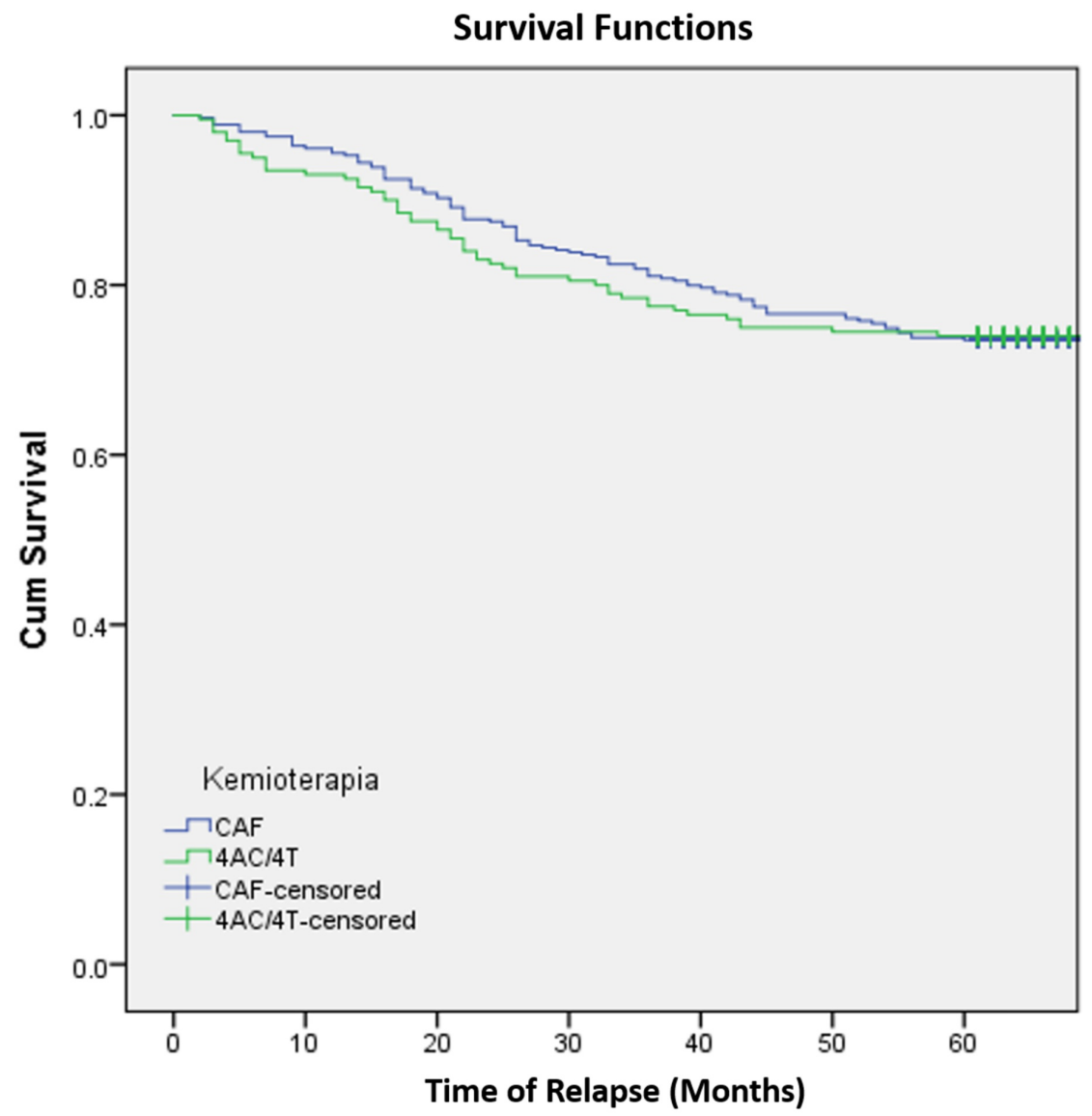

FIGURE 1: Kaplan-Meier curve for disease-free survival among patients with breast cancer based on adjuvant chemotherapy received

CAF: anthracycline-based adjuvant chemotherapy; AC/T: anthracycline-plus-taxane adjuvant chemotherapy 


\section{Cureus}

$\mathrm{AC} / \mathrm{T}$ was a little more efficient than CAF in avoiding relapse (74 \% vs. $73.5 \%$ ). However, there was no statistically significant difference between the two treatment regimens (p: 0.923).

The median DFS was higher for CAF than AC/T patients, but the total DFS was higher for the AC/T patient group (although the difference was not statistically significant; Breslow and Tarone-Ware tests, p: 0.758, p: 0.839 ; with a very high value of safety).

Table 2 displays the distribution of demographic and clinical characteristics, along with relapse data for each regimen. For this analysis, women were categorized into "no relapse at all" (AC/T, n = 148; CAF, $\mathrm{n}=$ 264) and "relapse" (AC/T n = 52; CAF, $\mathrm{n}=95)$.

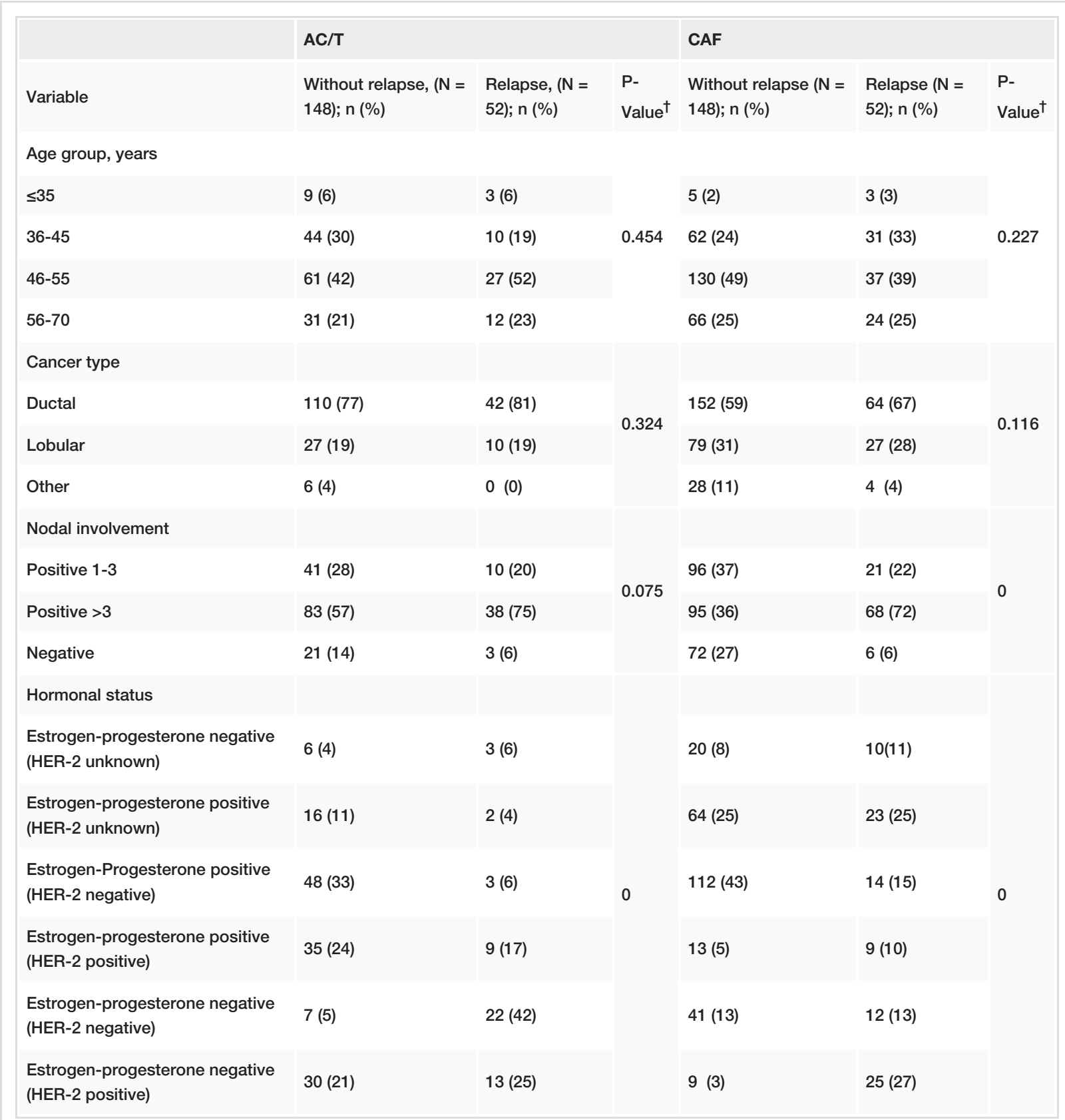

TABLE 2: Distribution of demographic and clinical characteristics by relapse status of Albanian woman with breast cancer treated with $\mathrm{AC} / \mathrm{T}$ or $\mathrm{CAF}$

AC/T: anthracycline-plus-taxane adjuvant chemotherapy; CAF: anthracycline-based adjuvant chemotherapy; HER2: human epidermal growth factor receptor 2

†Fisher's exact test 
For patients in the AC/T group, the spread of triple-negative hormone values was significantly higher among women who relapsed (42\%) compared to those with no relapse (5\%), but this difference was not statistically significant (p: 0.075).

For patients in the CAF group, the ratio of at least three positive nodules was significantly higher among women who relapsed (72\%) compared to their non-relapsing counterparts (36\%). The negative nodule ratio was significantly lower among women who relapsed (6\%) compared to their non-relapsing counterparts (27\%); this difference was statistically significant (p: 0.000).

\section{Discussion}

Studies in the last few decades have consistently shown that chemotherapy produces significantly better DFS and OS [21]. Multiple components determine the necessity for patients requiring adjuvant chemotherapy. These include, but are not limited to, the tumor size, molecular subtype, histology, and tumor grade. The axillary and regional lymph node status and the tumor hormone receptor expression are also important considerations [22]. The nodal status also plays a role with any nodal involvement lowering the survival rate at five years [23].

Three randomized trials reported significant improvement in DFS by adding taxanes to the anthracyclinesbased regimen in the adjuvant settings of node-positive breast cancer [14-16]. However, another three large randomized trials that included both node-negative and node-positive patients showed negative results in DFS improvement with a taxane-based regimen as adjuvant chemotherapy in breast cancer in node-negative patients [17-19].

This study supports the addition of a taxane drug as an adjuvant chemotherapy regimen in breast cancer treatment because the 5-year DFS was higher in the AC/T group, although the difference was not statistically significant. This result may have been due to the inclusion of patients regardless of their nodal status. Based on our results, identifying at least three positive nodules was associated with higher rates of relapse, which aligns with the findings reported by Miller et al. [24]. However, the prevalence of triple-negative hormonal values was much higher in AC/T treatment patients who relapsed compared to those who did not (42\% vs. $5 \%, \mathrm{p}:<0.001)$, and this change was very significant. This finding is the same as in those reported in another study where triple-negative breast cancer is considered an aggressive subtype for which the addition of taxanes to anthracycline-based regimens produced no survival benefit when compared to anthracyclinebased regimens [25].

Our study had some limitations. The small number of cases, especially of those treated with the AC/T regimen, rules out broader generalization. The small number of cases obliged us to include all patients regardless of their nodal status. This limitation prohibits the study from directly illustrating the advantage of adding taxanes in breast cancer adjuvant chemotherapy. However, our results align with our previous work and indicate a potential benefit; more extensive studies that evaluate the use of taxanes independent of patient nodal status are warranted. Properly evaluating the effectiveness of adding taxanes in therapy also requires an assessment of the toxicity and side effects of an $\mathrm{AC} / \mathrm{T}$ regimen, which this study could not do as we were limited by patient medical records that did not document toxicity and adverse side effects. Future prospective studies should evaluate the adverse side effects of each therapy and patient quality of life.

\section{Conclusions}

The AC/T regimen was slightly more efficacious than the CAF regimen for breast cancer patients in terms of avoiding relapse, but the difference was not statistically significant. Therefore, we can conclude that adding taxanes to adjuvant chemotherapy for women diagnosed with breast cancer is not beneficial for every subgroup, especially those with triple-negative breast cancer. Hence, the future of breast cancer therapy remains chemotherapy individualized for each patient for optimal outcomes.

\section{Additional Information}

\section{Disclosures}

Human subjects: Consent was obtained by all participants in this study. Animal subjects: All authors have confirmed that this study did not involve animal subjects or tissue. Conflicts of interest: In compliance with the ICMJE uniform disclosure form, all authors declare the following: Payment/services info: All authors have declared that no financial support was received from any organization for the submitted work. Financial relationships: All authors have declared that they have no financial relationships at present or within the previous three years with any organizations that might have an interest in the submitted work. Other relationships: All authors have declared that there are no other relationships or activities that could appear to have influenced the submitted work.

\section{Acknowledgements}

Acknowledgement for Prof. Vilma Papajani for the support during the research and on revising the work at the final process. 


\section{References}

1. Albanian Institute of Statistics: INSTAT health. (2018). Accessed: February 11, 2020: http://www.instat.gov.al/en/themes/social-condition/health.

2. Chew HK: Adjuvant therapy for breast cancer: who should get what? . West J Med. 2001, 174:284-287. 10.1136/ewjm.174.4.284

3. Tarifa D, Sallaku A, Lako S: Chemotherapy and the systemic cancer treatment (book in Albanian) . Ombra GVG, Tirana, Albania; 2007.

4. Bonadonna G, Brusamolino E, Valagussa P, et al.: Combination chemotherapy as an adjuvant treatment in operable breast cancer. N Engl J Med. 1976, 294:405-410. 10.1056/NEJM197602192940801

5. Levine MN, Pritchard KI, Bramwell VH, Shepherd LE, Tu D, Paul N: Randomized trial comparing cyclophosphamide, epirubicin, and fluorouracil with cyclophosphamide, methotrexate, and fluorouracil in premenopausal women with node-positive breast cancer: update of National Cancer Institute of Canada Clinical Trials Group Trial MA5. J Clin Oncol. 2005, 23:5166-5170. 10.1200/JCO.2005.09.423

6. Peto R, Davies C, Godwin J, et al.: Comparisons between different polychemotherapy regimens for early breast cancer: meta-analyses of long-term outcome among 100,000 women in 123 randomised trials. Lancet. 2012, 379:432-444. 10.1016/S0140-6736(11)61625-5

7. Ahmann DL, Bisel HF, Eagan RT, Edmonson JH, Hahn RG: Controlled evaluation of adriamycin (NSC123127) in patients with disseminated breast cancer. Cancer Chemother Rep. 1974, 58:877-882.

8. Jones S, Holmes FA, O'Shaughnessy J, et al.: Docetaxel with cyclophosphamide is associated with an overall survival benefit compared with doxorubicin and cyclophosphamide: 7-year follow-up of US Oncology Research Trial 9735. J Clin Oncol. 2009, 27:1177-1183. 10.1200/JCO.2008.18.4028

9. Henderson IC, Berry DA, Demetri GD, et al.: Improved outcomes from adding sequential paclitaxel but not from escalating doxorubicin dose in an adjuvant chemotherapy regimen for patients with node-positive primary breast cancer. J Clin Oncol. 2003, 21:976-983. 10.1200/JCO.2003.02.063

10. Martin M, Pienkowski T, Mackey J, et al.: Adjuvant docetaxel for node-positive breast cancer. N Engl J Med. 2005, 352:2302-2313. 10.1056/NEJMoa043681

11. Mackey JR, Martin M, Pienkowski T, et al.: Adjuvant docetaxel, doxorubicin, and cyclophosphamide in node-positive breast cancer: 10-year follow-up of the phase 3 randomised BCIRG 001 trial. Lancet Oncol. 2013, 14:72-80. 10.1016/S1470-2045(12)70525-9

12. Roché H, Fumoleau P, Spielmann M, et al.: Sequential adjuvant epirubicin-based and docetaxel chemotherapy for node-positive breast cancer patients: the FNCLCC PACS 01 Trial. J Clin Oncol. 2006, 24:5664-5671. 10.1200/JCO.2006.07.3916

13. Nitz U, Gluz O, Huober J, et al.: Final analysis of the prospective WSG-AGO EC-Doc versus FEC phase III trial in intermediate-risk (pN1) early breast cancer: efficacy and predictive value of Ki67 expression. Ann Oncol. 2014, 25:1551-1557. 10.1093/annonc/mdu186

14. Mamounas EP, Bryant J, Lembersky B, et al.: Paclitaxel after doxorubicin plus cyclophosphamide as adjuvant chemotherapy for node-positive breast cancer: results from NSABP B-28. J Clin Oncol. 2005, 23:3686-3696. 10.1200/JCO.2005.10.517

15. Martín M, Rodríguez-Lescure A, Ruiz A, et al.: Randomized phase 3 trial of fluorouracil, epirubicin, and cyclophosphamide alone or followed by paclitaxel for early breast cancer. J Natl Cancer Inst. 2008, 100:805814. 10.1093/jnci/djn151

16. Martín M, Seguí MA, Antón A, et al.: Adjuvant docetaxel for high-risk, node-negative breast cancer . N Engl J Med. 2010, 363:2200-2210. 10.1056/NEJMoa0910320

17. Goldstein LJ, O’Neill A, Sparano JA, Perez EA, Shulman LN, Martino S, Davidson NE: Concurrent doxorubicin plus docetaxel is not more effective than concurrent doxorubicin plus cyclophosphamide in operable breast cancer with 0 to 3 positive axillary nodes: North American Breast Cancer Intergroup Trial E 2197. J Clin Oncol. 2008, 26:4092-4099. 10.1200/JCO.2008.16.7841

18. Ellis P, Barrett-Lee P, Johnson L, et al.: Sequential docetaxel as adjuvant chemotherapy for early breast cancer (TACT): an open-label, phase III, randomised controlled trial. Lancet. 2009, 373:1681-1692. 10.1016/S0140-6736(09)60740-6

19. Burnell M, Levine MN, Chapman JA, et al.: Cyclophosphamide, epirubicin, and fluorouracil versus dosedense epirubicin and cyclophosphamide followed by paclitaxel versus doxorubicin and cyclophosphamide followed by paclitaxel in node-positive or high-risk node-negative breast cancer. J Clin Oncol. 2010, 28:7782. 10.1200/JCO.2009.22.1077

20. Hilaj E, Toska V, Shpati K: Relapse occurrence among Albanian women diagnosed with breast cancer and treated with taxane-based regimens as adjuvant chemotherapy. Albanian Med J. 2016, 1:17-21.

21. Berry DA, Cronin KA, Plevritis SK, et al.: Effect of screening and adjuvant therapy on mortality from breast cancer. N Engl J Med. 2005, 353:1784-1792. 10.1056/NEJMoa050518

22. Crozier JA, Swaika A, Moreno-Aspitia A: Adjuvant chemotherapy in breast cancer: to use or not to use, the anthracyclines. World J Clin Oncol. 2014, 5:529-538. Accessed: February 27, 2020: https://www.ncbi.nlm.nih.gov/pmc/articles/PMC4127622/. 10.5306/wjco.v5.i3.529

23. Cheng L, Swartz MD, Zhao H, et al.: Hazard of recurrence among women after primary breast cancer treatment--a 10-year follow-up using data from SEER-Medicare. Cancer Epidemiol Biomarkers Prev. 2012, 21:800-809. 10.1158/1055-9965.EPI-11-1089

24. Roberts MC, Miller DP, Shak S, Petkov VI: Breast cancer-specific survival in patients with lymph nodepositive hormone receptor-positive invasive breast cancer and Oncotype DX Recurrence Score results in the SEER database. Breast Cancer Res Treat. 2017, 163:303-310. 10.1007/s10549-017-4162-3

25. Lakshmaiah KC, Anand A, Babu KG, et al.: Role of taxanes in triple-negative breast cancer: a study from tertiary cancer center in South India. World J Oncol. 2017, 8:110-116. 10.14740/wjon1054w 